\title{
Algal biomass conversion to bioethanol - a step-by-step assessment
}

\begin{abstract}
The continuous growth in global population and the ongoing development of countries such as China and India have contributed to a rapid increase in worldwide energy demand. Fossil fuels such as oil and gas are finite resources, and their current rate of consumption cannot be sustained. This, coupled with fossil fuels' role as pollutants and their contribution to global warming, has led to increased interest in alternative sources of energy production. Bioethanol, presently produced from energy crops, is one such promising alternative future energy source and much research is underway in optimizing its production. The economic and temporal constraints that crop feedstocks pose are the main downfalls in terms of the commercial viability of bioethanol production. As an alternative to crop feedstocks, significant research efforts have been put into utilizing algal biomass as a feedstock for bioethanol production. Whilst the overall process can vary, the conversion of biomass to bioethanol usually contains the following steps: (i) pretreatment of feedstock; (ii) hydrolysis; and (iii) fermentation of bioethanol. This paper reviews different technologies utilized in the pretreatment and fermentation steps, and critically assesses their applicability to bioethanol production from algal biomass. Two different established fermentation routes, single-stage fermentation and two-stage gasification/fermentation processes, are discussed. The viability of algal biomass as an alternative feedstock has been assessed adequately, and further research optimisation must be guided toward the development of cost-effective scalable methods to produce high bioethanol yield under optimum economy.
\end{abstract}

Keyword: Algae; Bioethanol; Fermentation; Gasification; Hydrolysis 